\title{
Skillful multiyear predictions of ocean acidification in the California Current
}

\section{System}

Riley X. Brady ${ }^{1 *}$, Nicole S. Lovenduski ${ }^{1}$, Stephen G. Yeager ${ }^{2}$, Matthew C. Long ${ }^{2}$, Keith Lindsay $^{2}$

${ }^{1}$ Department of Atmospheric and Oceanic Sciences and Institute of Arctic and Alpine Research, University of Colorado, Boulder, Colorado, USA

${ }^{2}$ Climate and Global Dynamics Laboratory, National Center for Atmospheric Research, Boulder, Colorado, USA

${ }^{*}$ Correspondence to: riley.brady@colorado.edu

1 Abstract: The California Current System (CCS) sustains economically valuable fisheries and is particularly vulnerable to ocean acidification, due to its natural upwelling of carbon-enriched waters that generate corrosive conditions for local ecosystems. Here we use a novel suite of retrospective, initialized ensemble forecasts with an Earth system model (ESM) to predict the evolution of surface $\mathrm{pH}$ anomalies in the CCS. We show that the forecast system skillfully predicts observed surface $\mathrm{pH}$ variations a year in advance over a naïve forecasting method, with the potential for skillful prediction up to five years in advance. Skillful predictions of surface $\mathrm{pH}$ are mainly derived from the

9 initialization of dissolved inorganic carbon anomalies that are subsequently transported

10 into the CCS. Our results demonstrate the potential for ESMs to provide predictions

11 relevant to managing the onset and impacts of ocean acidification on large scales in the 
12 CCS. Initialized ESMs could also provide boundary conditions to improve high-

13 resolution regional forecasting systems.

\section{Introduction}

15 Ocean acidification is an ongoing large-scale environmental problem, whereby the

16 absorption of anthropogenic $\mathrm{CO}_{2}$ by the ocean lowers its $\mathrm{pH}$, impacting ocean

17 ecosystems worldwide ${ }^{1}$. The California Current System (CCS) supports productive

18 fisheries crucial to the US economy and is particularly vulnerable to ocean acidification

19 due to the upwelling of naturally corrosive (i.e., relatively low $\mathrm{pH}$ ) waters to the surface?

20 The upwelling process results from equatorward winds along the western North

21 American coastline. These winds facilitate both coastal upwelling and curl-driven Ekman

22 suction, forcing waters enriched in carbon and nutrients from beneath the thermocline to

23 the surface ${ }^{3}$. These nutrient subsidies drive high productivity in CCS waters, essential to

24 supporting regional fisheries ${ }^{4}$. However, the upwelled waters are also corrosive due to

25 their high remineralized carbon content. The air-to-sea flux of anthropogenic $\mathrm{CO}_{2}$ into

26 the CCS further compounds this natural acidity. Multiple studies over the past decade

27 have observed coastal CCS waters that are anomalously low in surface $\mathrm{pH}$ relative to

28 the historical state of the system and undersaturated with respect to calcium carbonate

29 minerals ${ }^{5-7}$. These conditions adversely affect a wide range of organisms that

30 precipitate calcium carbonate shells, such as pteropods, coccolithophores, and

31 shellfish ${ }^{1}$. Shellfish in particular contribute significantly to the $\$ 6 \mathrm{~B}$ in revenue per year

32 provided by commercial and recreational fisheries in the CCS ${ }^{8}$. The CCS's intersection 
33 between economically valuable fisheries and natural vulnerability to ocean acidification

34 makes it a high-priority region to study for multiyear biogeochemical predictions.

\section{Results}

\section{Forecasting ocean biogeochemistry in the California Current}

Prediction efforts for the CCS have focused primarily on using seasonal forecasts of sea surface temperature ${ }^{9-11}$ (SST) and biogeochemical variables ${ }^{12}$ (e.g., dissolved oxygen and bottom $\mathrm{pH}$ ) as inputs into ecosystem forecasting models. A more recent effort demonstrates the potential for skillful initialized predictions of surface chlorophyll in the CCS with two year forecasts ${ }^{13}$. However, no studies have attempted to predict ocean biogeochemistry in the CCS at the multiannual to decadal scale, as decadal forecasting of ocean biogeochemistry is still in its infancy ${ }^{14-18}$. This temporal scale is critical for fisheries managers, as it aids them in setting annual catch limits, changing and introducing closed areas, and adjusting quotas for internationally shared fish stocks ${ }^{19}$.

Some level of skill can be provided by persisting anomalies from year to year in the system ${ }^{19}$. These so-called persistence forecasts are commonly used as a reference to put initialized skill into context and work at lead times commensurate with the decorrelation timescales of the system ${ }^{9-11,19}$. On the other hand, initialized predictions use a physically based modeling framework to advance information from initial conditions forward in time; if the system is predictable (i.e., sufficiently deterministic) and the model skillful, this can yield a powerful forecasting framework. Ensemble simulations of initialized ESMs provide the most powerful approach currently available for improving upon decadal persistence forecasts. Their coupling of global physical 
55 models of the atmosphere, ocean, cryosphere, and land with the carbon cycle,

terrestrial and marine ecosystems, atmospheric chemistry, and natural and human disturbances allows one to deeply investigate how interactions between the physical climate system and biosphere lead to predictability in a complex system such as the $\operatorname{CCS}^{20}$. These predictions have the potential to improve upon persistence forecasts, pushing the horizon of forecasting ecosystem stressors past a single season or year.

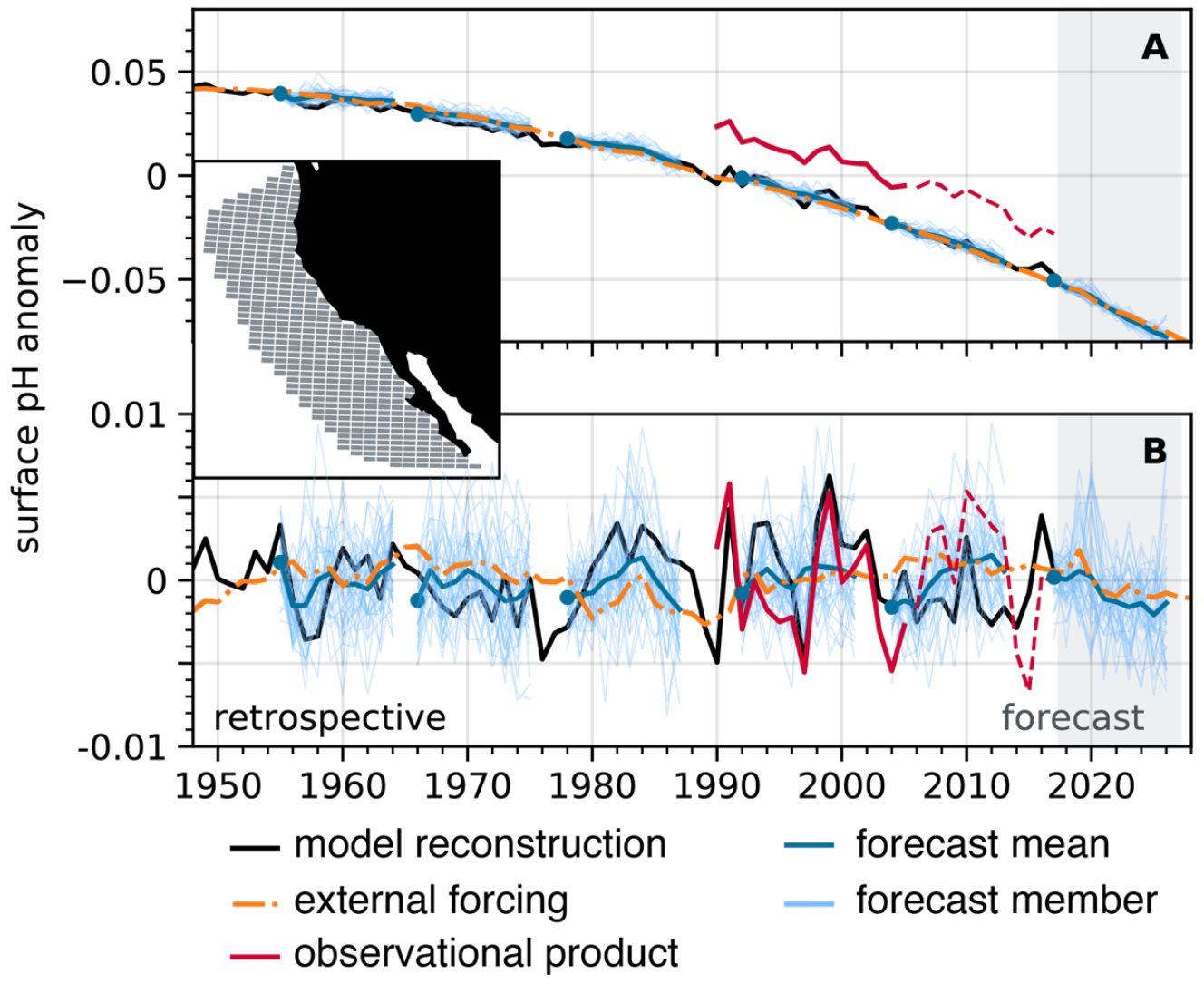

Fig. 1. CESM-DPLE experimental design and near-future surface $\mathrm{pH}$ anomaly forecast. (A) Trended and (B) detrended area-weighted annual surface $\mathrm{pH}$ anomalies for the (black) reconstruction, (red) observational product, (orange) CESM-LE ensemble mean, and (blue) CESM-DPLE decadal forecasts initialized in 1954, 1965, 1977, 1991, 2003, and 2017 (other initializations were omitted for visual clarity). The dark blue line is the ensemble mean forecast, and thin blue lines are the individual 40 forecasts. The blue dots do not sit exactly atop the black line due to the rapid divergence of forecasts away from 
initialization within weeks. The dashed red lines denote when the model loses observed variability in atmospheric $\mathrm{CO}_{2}$ forcing (see Fig. $\mathrm{S} 1 \mathrm{~A}$ ). The inset shows the California Current large Marine Ecosystem bounds, over which all area-weighted analyses are computed.

Here we use an initialized global ESM with embedded ocean biogeochemistry, the Community Earth System Model Decadal Prediction Large Ensemble ${ }^{21}$ (CESMDPLE), to make retrospective forecasts of surface pH anomalies in the CCS from 1955 through 2017. The CESM-DPLE employs an ocean model with nominal $1^{\circ} \times 1^{\circ}$ horizontal resolution and 60 vertical levels. Forty ensemble members were initialized annually on November $1^{\text {st }}$ from a forced ocean-sea ice reconstruction (hereafter referred to as the "reconstruction") and then the coupled simulations were integrated forward for ten years (Fig. 1, A and B; see methods and supplementary). The reconstruction is skillful in representing surface $\mathrm{pH}$ variability on seasonal to interannual timescales in the CCS (Fig. 2). Due to the diverse terminology used in weather and climate forecasting ${ }^{22}$, we are careful with our definitions. We use the phrase "potential predictability" when referring to correlations between CESM-DPLE and the reconstruction. High correlation coefficients (i.e., high potential predictability) represent the theoretical upper limit for predictions in the real world, given the chaotic nature of the climate system ${ }^{23}$. We use the phrase "predictive skill" when comparing CESM-DPLE to observations; skill demonstrates our ability to predict the true evolution of the real world with CESM-DPLE. We quantify our ability to predict anomalies with the anomaly correlation coefficient (ACC), and our accuracy in predicting anomaly magnitudes with the normalized mean absolute error (NMAE; see methods). We compare our initialized forecasts to a simple persistence forecast and the uninitialized CESM Large Ensemble ${ }^{24}$ (CESM-LE) mean, 
91 which includes the same external forcing (i.e., rising atmospheric $\mathrm{CO}_{2}$ ) as the CESM-

92 DPLE. The former assesses whether CESM-DPLE is useful relative to a simple

93 forecasting method, while the latter determines the degree to which initialization

94 engenders predictability beyond that afforded by supplying the model with time-varying

95 forcing. We test predictive skill by comparing the initialized forecasts to a gridded

96 observational product of surface $\mathrm{pH}$ from the Japan Meteorological Agency (JMA),

97 which spans 1990-201725,26. This product is based upon empirical relationships derived

98 for alkalinity and $\mathrm{pCO}_{2}$ as functions of in situ measurements, such as SST and sea

99 surface height, which were then used in a carbonate system solver to derive gridded

100 surface $\mathrm{pH}$ (see methods). Our focus in this study is on surface $\mathrm{pH}$ anomalies within the

101 California Current Large Marine Ecosystem (see the inset in Fig. 1 for the spatial

102 domain). We focus on the entire Large Marine Ecosystem, since the $1^{\circ} \times 1^{\circ}$ model grid

103 cannot resolve the coastal upwelling of corrosive waters that occurs on scales smaller

104 than the grid resolution. We remove a second-order polynomial fit from all surface $\mathrm{pH}$

105 time series, since the long-term ocean acidification signal dominates over the 1955-

1062017 hindcast period (Fig. 1A). We aim to test our ability to predict year-to-year

107 variations in CCS surface pH anomalies (Fig. 1B), which act to temporarily accelerate or

108 slow down the ongoing ocean acidification trend. 


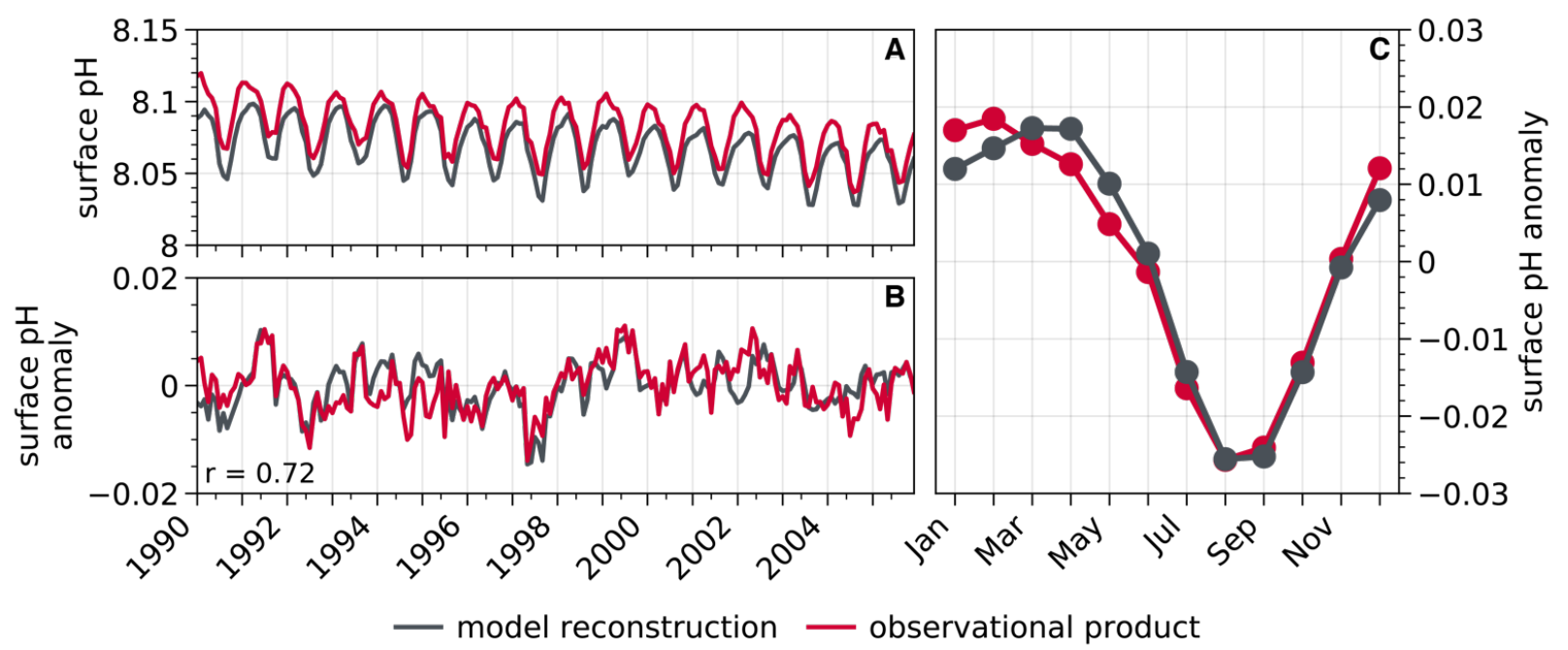

Fig 2. Area-weighted temporal evaluation of surface $\mathrm{pH}$ in the model reconstruction. (A) Monthly surface $\mathrm{pH}$ in the California Current over 1990-2005 for the model reconstruction (black) and observational product (red). (B) As in (A), but for anomalies after removing a second-order polynomial fit and the seasonal cycle. The correlation coefficient between the observational product and model reconstruction is shown in the bottom left of (B). (C) As in the other panels, but for the mean monthly seasonal cycle over 1990-2005.

\section{Model Evaluation}

Previous evaluations of the physical circulation and carbonate chemistry in the version of CESM used for CESM-DPLE suggest that, despite the relatively coarse $1^{\circ} \times 1^{\circ}$ model grid, CESM provides a good fit to observational climatologies of alongshore wind stress, surface $\mathrm{pCO}_{2}$, and air-sea $\mathrm{CO}_{2}$ fluxes in the $\mathrm{CCS}^{27,28}$. Modeled alongshore wind stress - the primary driver of coastal upwelling-closely matches the magnitude and seasonality of observations, with peak upwelling-favorable conditions spanning April to September ${ }^{27}$. The large-scale spatial structure of air-sea $\mathrm{CO}_{2}$ fluxes in the model exhibits poleward $\mathrm{CO}_{2}$ uptake and equatorward $\mathrm{CO}_{2}$ outgassing, matching that of modern observationally based estimates ${ }^{28,29}$. Importantly, we note that CESM cannot 
126 capture the nearshore outgassing of $\mathrm{CO}_{2}$ associated with the coastal upwelling of

127 carbon-enriched waters that occurs on a scale smaller than the resolution of the model

128 grid $^{28,30}$. The modeled monthly climatology of area-weighted surface ocean $\mathrm{pCO}_{2}$ in the

129 CCS closely resembles that of the observationally based estimate, due to the model's

130 proper simulation of the magnitude and phasing of thermal (solubility-driven) and non-

131 thermal (circulation- and biology-driven) $\mathrm{pCO}_{2}$ effects ${ }^{28,29}$.

We further evaluate the carbonate chemistry of the CCS region in CESM-DPLE by comparing surface ocean $\mathrm{pH}$ from our reconstruction with the gridded JMA

134 observational $\mathrm{pH}$ product ${ }^{25,26}$. We limit the evaluation period to $1990-2005$, as the JMA

135 observational product begins in 1990 , and the reconstruction is forced using non-

136 historical atmospheric $\mathrm{CO}_{2}$ from 2006 onwards (Fig S1). Over the 1990-2005 period, the

137 spatial distribution of $\mathrm{pH}$ climatologies in the reconstruction closely match that of the

138 observational product, with both suggesting higher surface $\mathrm{pH}$ during the wintertime

139 downwelling season and lower surface $\mathrm{pH}$ in the summertime upwelling season (Fig.

140 S2, see also Fig. 2C). High-resolution model solutions demonstrate similar spatial

141 patterns and seasonality of surface $\mathrm{pH}$ in this region ${ }^{31}$. The reconstruction has a slight

142 acidic bias (Fig. $2 A)$, with a relative mean bias in the hydrogen ion concentration $([\mathrm{H}+])$

143 ranging from $2.9 \%$ to $4.2 \%$ across the CCS (Fig. S2, I to L). Over the area-weighted

$144 \mathrm{CCS}$ (Fig. 2), the reconstruction simulates a linear change in $\mathrm{pH}$ of -0.026 over the

145 1990-2005 period, compared to the observational product's linear change of -0.029

146 (Fig. 2A). Both the reconstruction and observational product exhibit an interannual

147 standard deviation of 0.003 in surface $\mathrm{pH}$. Thus, the interannual variability in both the 
148 model and observations is between 1.5 to 2 times greater than the ocean acidification

149 trend over the course of one year. Surface $\mathrm{pH}$ anomalies in both the reconstruction and

150 observational product exhibit a decorrelation time scale of four months (Fig. S3). The

151 reconstruction closely replicates $\mathrm{pH}$ monthly anomalies (second-order polynomial fit and

152 seasonal cycle removed) from the JMA observational product (Fig. 2B), with a linear

153 correlation coefficient of 0.72 .

We identify the drivers of reconstructed surface $\mathrm{pH}$ variability in the CCS by

155 estimating the contributions from variations in salinity, alkalinity, SST, and dissolved

156 inorganic carbon (DIC; see methods). The two major terms driving variability in surface

$157 \mathrm{pH}$ are DIC and SST, whose standard deviation is approximately three times that of

158 surface $\mathrm{pH}$ (Fig. S4). These two terms exhibit low-frequency variability and are

159 significantly correlated with modes of variability such as the Pacific Decadal Oscillation

160 (PDO) and El Niño-Southern Oscillation (ENSO). The linear correlation coefficient

161 between DIC and SST residuals and the PDO is 0.66 and 0.73 , and ENSO is 0.52 and

1620.64 , respectively (Table $\mathrm{S} 1$ ). Since surface $\mathrm{pH}$ is the small residual of many variables,

163 it has a correlation coefficient of nearly zero with both modes of variability (Table S1). 

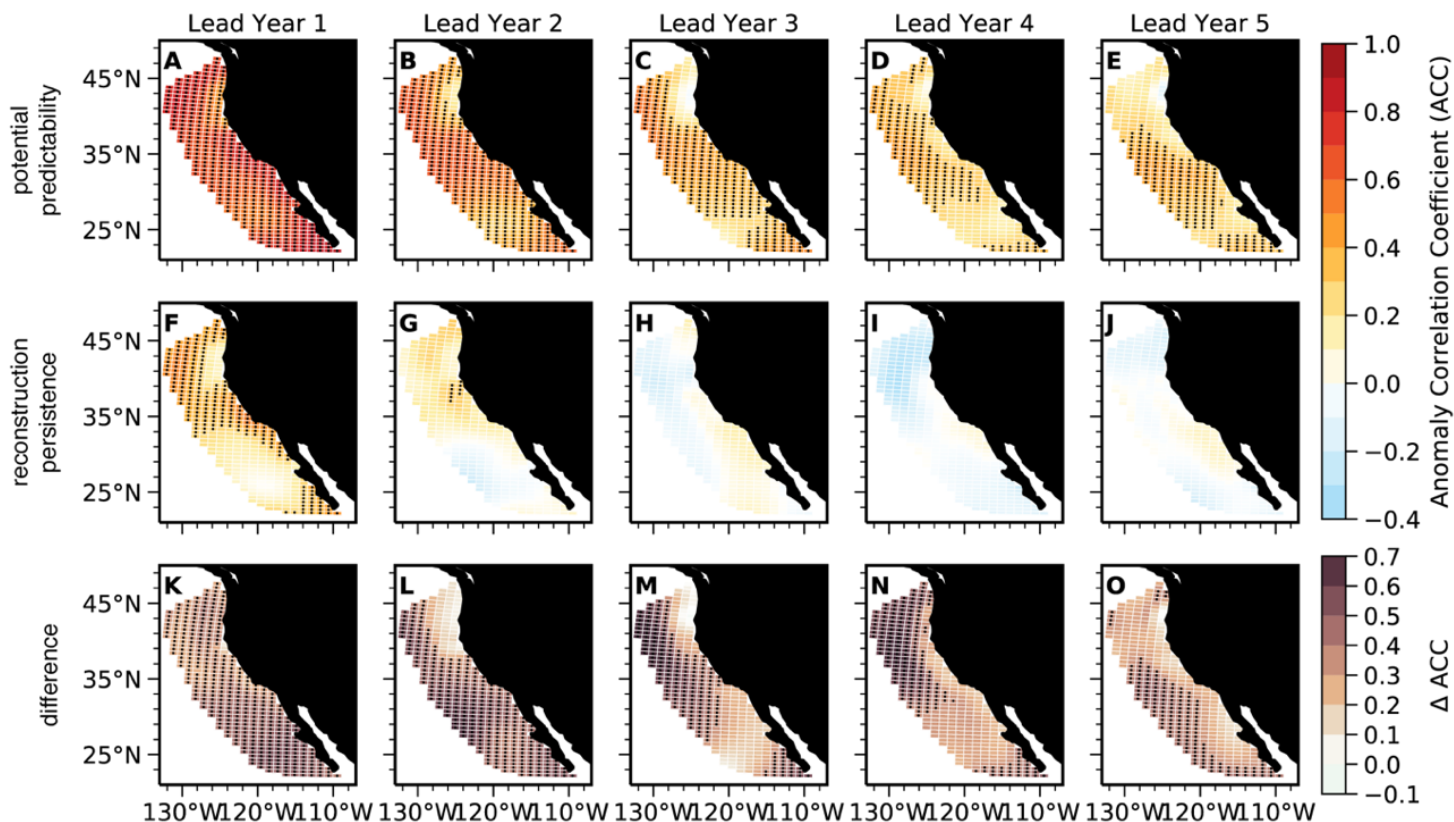

164

165

166

Fig. 3. Potential predictability of surface $\mathrm{pH}$ in the California Current. (A to E) CESM-DPLE initialized forecast of detrended annual surface $\mathrm{pH}$ anomalies for lead years one through five correlated with the reconstruction. ( $\mathbf{F}$ to $\mathbf{J})$ Persistence forecast for the reconstruction for lead years one through five.

Stippling in $\mathbf{A}$ to $\mathbf{J}$ denotes statistically significant correlations at the $95 \%$ level using a $t$ test. An effective sample size is used in the $t$ test to account for autocorrelation in the two time series being correlated. (K to 0) Difference between the CESM-DPLE forecast ACCs and persistence. Stippling indicates that the initialized prediction is statistically significant over the persistence forecast at the $95 \%$ level using a $z$ test. Only positive ACCs and $\triangle \mathrm{ACCs}$ are stippled.

\section{Predictions of simulated and observed surface $\mathrm{pH}$}

Retrospective forecasts of detrended annual surface $\mathrm{pH}$ anomalies in the CCS suggest a potential to predict surface $\mathrm{pH}$ up to five years in advance over a simple persistence forecast (Figs. 3 and 4). Although a persistence forecast is valuable at lead year one in parts of the CCS (Fig. 3F), the initialized forecast is statistically significant over persistence nearly everywhere (Fig. 3K). By lead year two, persistence begins to yield negative ACCs in the southern portion of the CCS, while retaining some positive 
180 correlation in the north; ACCs become non-significant and weakly negative from lead

181 year three and beyond (Fig. 3, $\mathrm{H}$ to J). The initialized forecast, in contrast, retains

182 predictability in the central and southern CCS through lead year five (Fig. 3, A to E).

183 Initialized predictions have higher ACCs $(\triangle A C C)$ than a persistence forecast

184 everywhere out to five-year leads, save for three coastal grid cells along the coastal

185 Pacific Northwest in lead year three (Fig. 3, $\mathrm{K}$ to O). An area-weighted perspective of

186 the CCS reveals that the initialized forecast is statistically significant over both

187 persistence and the uninitialized forecast through five-year leads (Fig. 4A). The lead

188 year one ACC of 0.72 explains over $50 \%$ of the variance in predicted surface $\mathrm{pH}$

189 anomalies and is comparable or better than the skill achieved by seasonal forecasts of

190 SSTs in the CCS $^{910}$. The NMAE is smaller than both persistence and the uninitialized

191 forecast over all ten lead years, and falls within the magnitude of surface $\mathrm{pH}$ interannual

192 variability in the model reconstruction (Fig. 4B). 

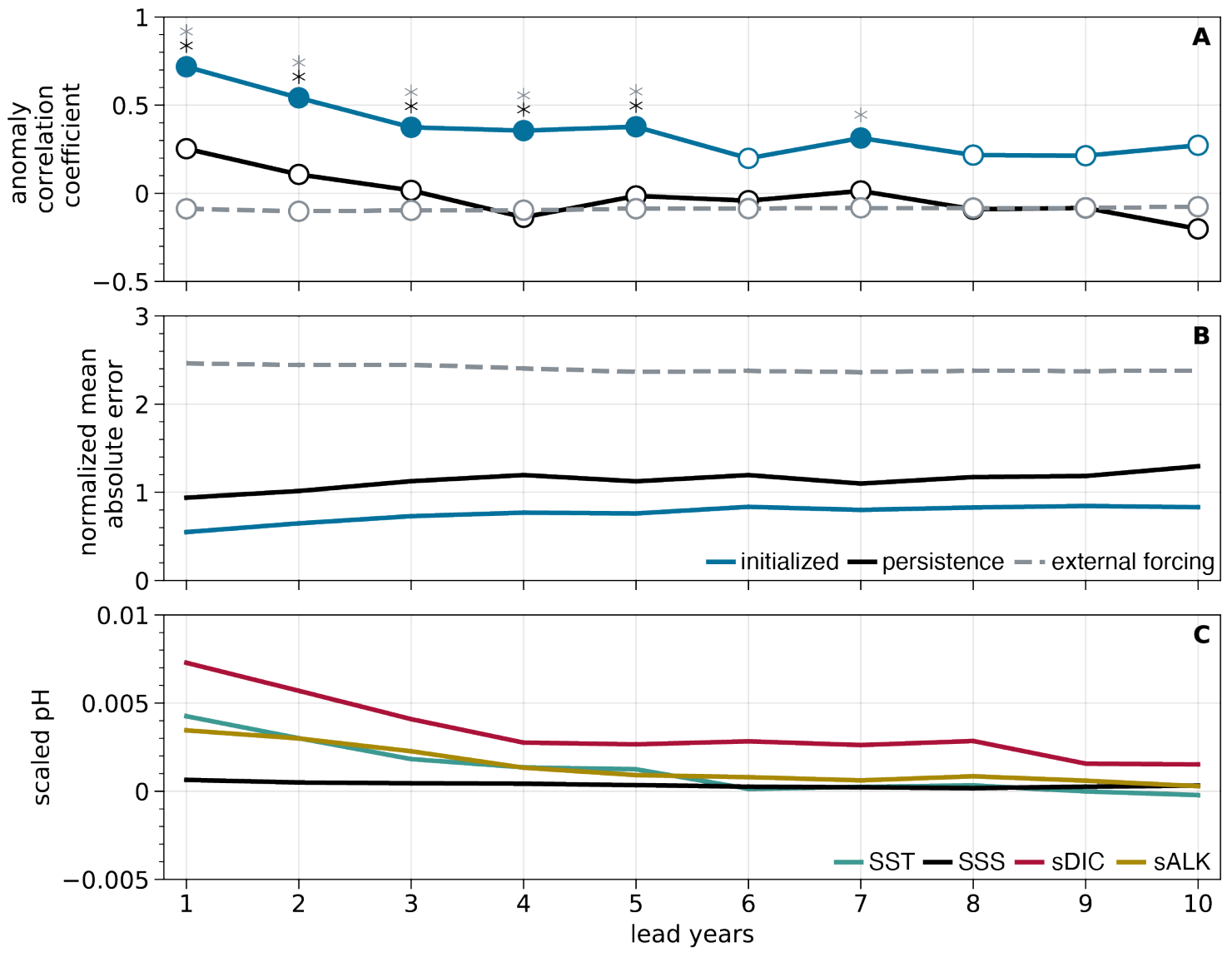

193

Fig. 4. Area-weighted potential predictability of surface $\mathrm{pH}$ in the California Current and driver variables of surface $\mathrm{pH}$ predictability. (A) ACCs for ten lead years for (blue) CESM-DPLE, (black) a persistence forecast from the reconstruction, and (grey) the uninitialized CESM-LE ensemble mean. Filled circles denote statistically significant positive correlations at the $95 \%$ level using a $t$ test. An effective sample size is used in the $t$ test to account for autocorrelation in the two time series being correlated. The critical value required for a statistically significant correlation ranges from 0.26 to 0.32 across leads, as computed by inverting the $t$ statistic formula. Black and gray asterisks indicate significant predictability over persistence and the uninitialized forecast at the 95\% level using a $z$ test, respectively. (B) As in (A), but for NMAE and without significance testing. Values below (above) one indicate that the forecast falls within (outside of) the interannual variability of surface $\mathrm{pH}$ in the reconstruction. (C) Scaled predictability in common $\mathrm{pH}$ units (see supplementary methods) of (black) sea surface salinity, (teal) sea surface temperature, (gold) salinity-normalized alkalinity, and (red) salinity-normalized dissolved inorganic carbon. 
Because the reconstruction simulates the mean state, seasonal cycle, and

variability of surface $\mathrm{pH}$ in the CCS well (Figs. 2 and S2), potential predictability extends to predictive skill relative to the observational product (Fig. 5). Initialized predictions have positive ACCs throughout most of the CCS at lead year one (Fig. 5A), and exhibit skill over persistence through lead year four from Cape Mendocino to Baja California

(Fig. $5, \mathrm{~K}$ to $\mathrm{N}$ ). Persistence in the observationally based surface $\mathrm{pH}$ estimate is somewhat useful south of Cape Mendocino at lead year one, but yields negative ACCs from lead years two to five throughout most of the CCS (Fig. 5, F to J). Note, however, that none of these correlations are statistically significant at the $95 \%$ level. Across all five lead years, ACCs from the initialized predictions are larger than those of observational persistence for most of the CCS (Fig. 5, $\mathrm{K}$ to $\mathrm{O}$ ), with an area-weighted mean $\triangle \mathrm{ACC}$ (the difference between ACCs for the initialized ensemble and observational product) ranging from 0.04 to 0.43 . Skill is lost for the southernmost portion of the CCS by lead year two (Fig. 5B), followed by the Pacific Northwest at lead year three (Fig. 5C). Mean absolute error in the initialized predictions of the observed surface $\mathrm{pH}$ are smaller than that of observational persistence for most of the CCS over five lead years (Fig. 6, K to O), and primarily falls within the magnitude of surface $\mathrm{pH}$ interannual variability in the observations (Fig. 6, A to E). Our results suggest that CESM-DPLE could be used for multiyear forecasting of surface $\mathrm{pH}$ variability in the CCS today. 

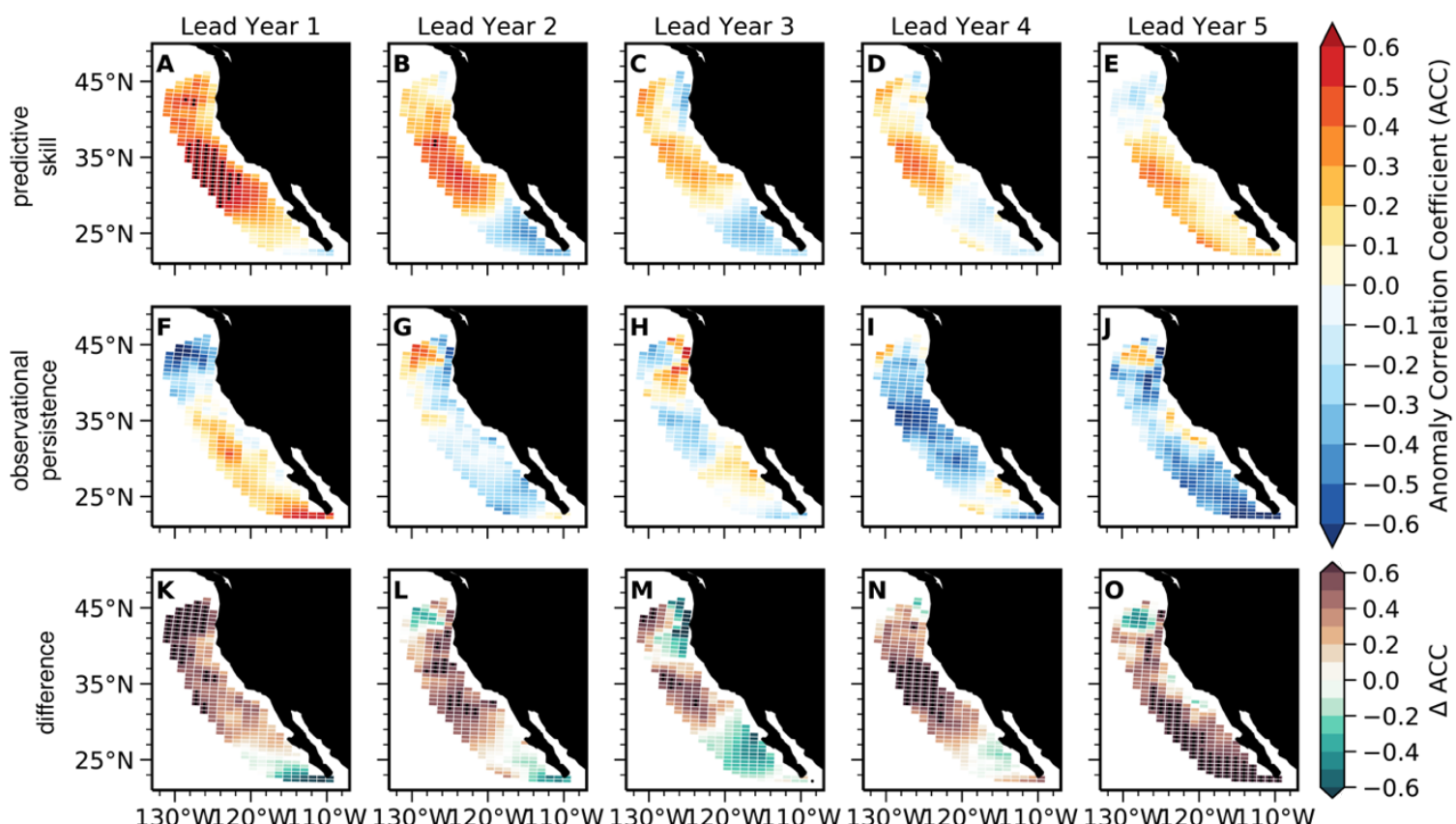

Fig. 5. Predictive skill of initialized surface $\mathrm{pH}$ anomaly forecasts relative to observations in the California

228 Current. (A to E) CESM-DPLE initialized forecast of detrended annual surface pH anomalies for lead

229 years one through five correlated with the observational product over 1990-2005. (F to J) Persistence

230 forecast for the observations for lead years one through five. Stippling in $\mathbf{A}$ to $\mathbf{J}$ denotes statistically

231 significant correlations at the $95 \%$ level using a $t$ test. An effective sample size is used in the $t$ test to

232 account for autocorrelation in the two time series being correlated. ( $\mathbf{K}$ to $\mathbf{O})$ Difference between the

233 CESM-DPLE forecast ACCs and observational persistence. Stippling indicates that the initialized

234 prediction is statistically significant over the observational persistence forecast at the $95 \%$ level using a $z$

235 test. Only positive ACCs and $\triangle \mathrm{ACC}$ s are stippled. 

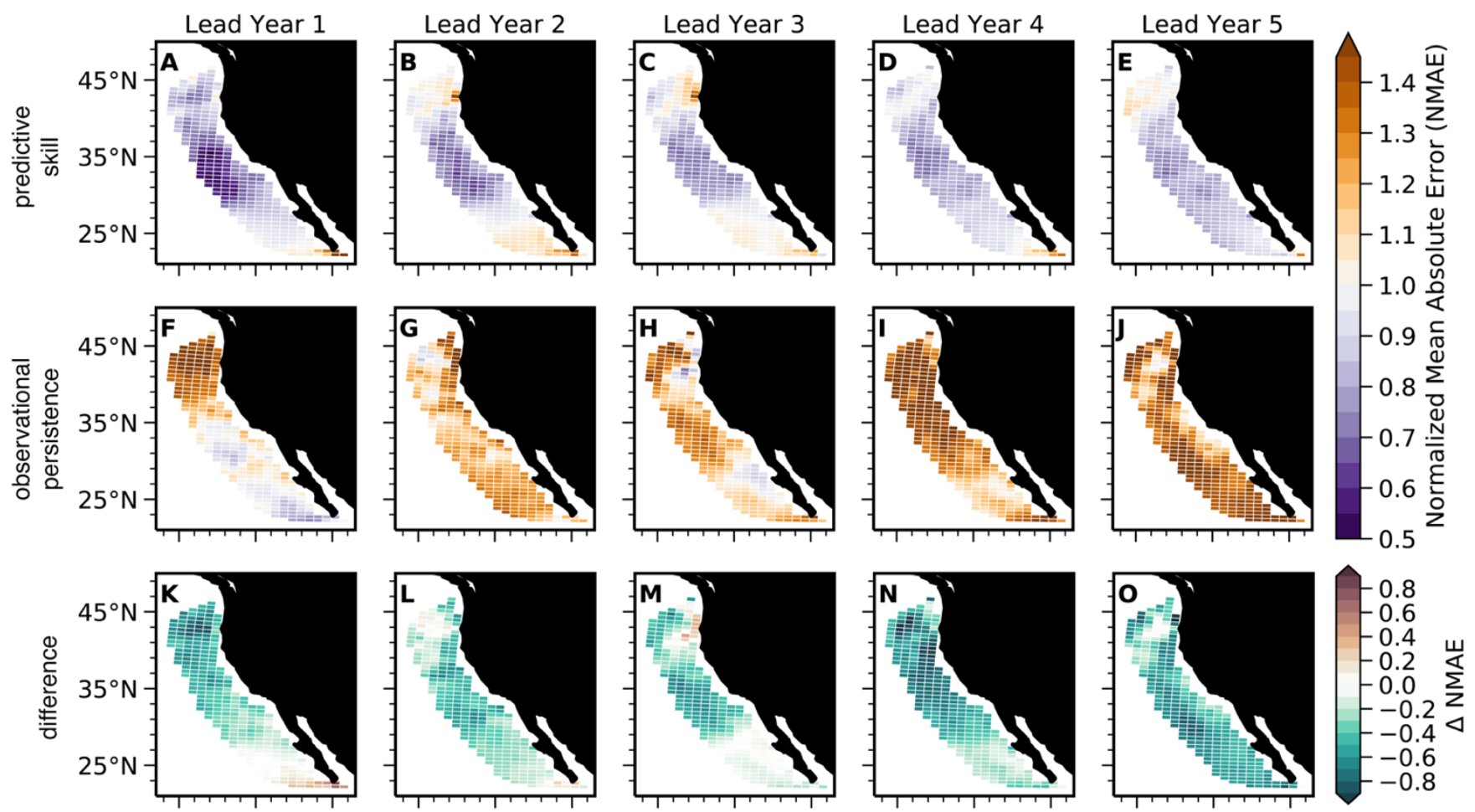

$130^{\circ} \mathrm{W} 120^{\circ} \mathrm{W} 110^{\circ} \mathrm{W} 130^{\circ} \mathrm{W} 120^{\circ} \mathrm{W} 110^{\circ} \mathrm{W} 130^{\circ} \mathrm{W} 120^{\circ} \mathrm{W} 110^{\circ} \mathrm{W} 130^{\circ} \mathrm{W} 120^{\circ} \mathrm{W} 110^{\circ} \mathrm{W} 130^{\circ} \mathrm{W} 120^{\circ} \mathrm{W} 110^{\circ} \mathrm{W}$

Fig. 6. Normalized mean absolute error of initialized surface $\mathrm{pH}$ anomaly forecasts relative to observations in the California Current. (A to E) NMAE of CESM-DPLE initialized forecast of detrended annual surface $\mathrm{pH}$ anomalies for lead years one through five relative to the observational product over 1990-2005. ( $\mathbf{F}$ to J) NMAE of a persistence forecast for the observations for lead years one through five.

241 Purple colors (values below one) indicate that the forecast error is smaller than the interannual variability

242 of observations; orange colors (values above one) indicate that the forecast error is larger than the

243 interannual variability of observations. ( $\mathbf{K}$ to $\mathbf{0}$ ) Difference between the CESM-DPLE forecast and

244 observational persistence NMAEs. Green colors indicate that the initialized forecasts have lower error

245 than the persistence forecast.

\section{Mechanisms of $\mathrm{pH}$ predictability}

247 We are further interested in what lends predictability to surface $\mathrm{pH}$ in the CCS. We

248 begin by investigating predictability in the driver variables of $\mathrm{pH}$ : SST, salinity, DIC, and 
249 alkalinity. By scaling these variables to common pH units (see methods), we can

250 deduce which drivers aid the most in predicting surface $\mathrm{pH}$. We find that predictability in

251 salinity-normalized DIC (sDIC) has the largest influence on surface $\mathrm{pH}$ predictability

252 over all ten lead years (Fig. 4C). The combined predictability of both SSTs and salinity-

253 normalized alkalinity is roughly equivalent to SDIC over the first five lead years, while

254 sea surface salinity plays a negligible role over all ten lead years (Fig. 4C). Predictability

255 in SDIC is mainly driven by the persistence of its anomalies, but is enhanced further by

256 initializations (Fig. S5). A budget analysis of DIC in the upper 150m of the CCS

257 suggests that variability in vertical and lateral DIC advection plays a leading role in

258 setting the DIC inventory (Fig. 7), as evidenced by the high correlation between the

259 advective flux and total tendency terms $(r=0.9)$. Source waters for the CCS exhibit

260 substantial interannual to decadal variability and are mainly comprised of subarctic

261 waters transported by the California Current (upper $200 \mathrm{~m}$ ) and eastern tropical Pacific

262 waters transported by the California Undercurrent (200-300 m), which propagate

263 biogeochemical anomalies into the system ${ }^{32,33}$. Thus, the subsurface and basin-wide

264 initializations of DIC-as well as predictability of meridional and vertical transport

265 variability -are crucial factors in making skillful multiyear predictions of surface $\mathrm{pH}$

266 variability. In turn, enhanced observations or reanalysis of these fields would be

267 necessary for operational forecasting of surface $\mathrm{pH}$ in the CCS. 
268

269

270

271

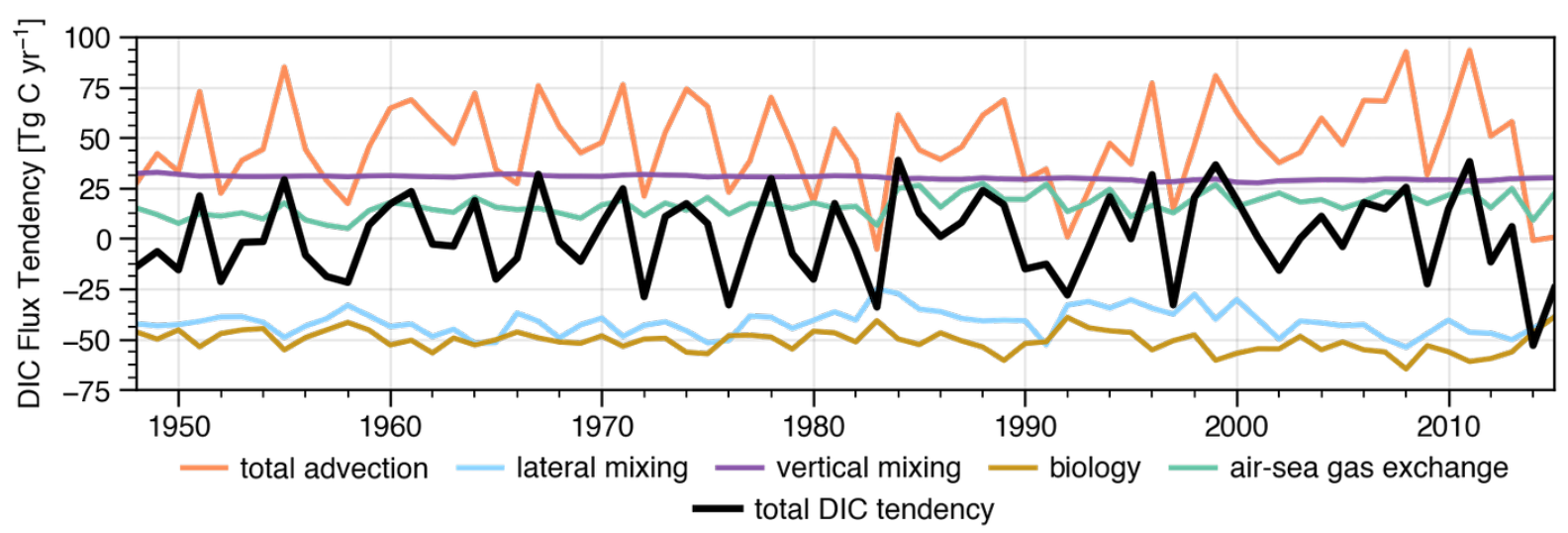

Fig 7. Dissolved inorganic carbon (DIC) budget of the California Current over the upper 150m. Time series of the individual annual tendency terms of DIC in the reconstruction integrated over the CCS laterally and to $150 \mathrm{~m}$ vertically (the approximate mean mixed layer depth in the model reconstruction).

The colored lines show the individual terms, while the black line shows the total integrated DIC tendency.

\section{Discussion}

While this study presents a very promising first result, there are some caveats worth noting. Simulations were run with a spatial resolution of approximately $100 \mathrm{~km} \times 100$ $\mathrm{km}$. In turn, we do not explicitly resolve the fine-scale coastal upwelling of corrosive waters (which occurs within roughly $30 \mathrm{~km}$ of the coastline in the CCS), but instead simulate the combined effect of coastal and curl-driven upwelling in nearshore grid cells ${ }^{27}$. Our simulation also uses subgrid scale parameterizations to capture the important process of eddy-induced offshore flux of tracers in the $\mathrm{CCS}^{34,35}$. Despite the coarse resolution, alongshore winds, upwelling, air-sea $\mathrm{CO}_{2}$ fluxes, surface $\mathrm{pCO}_{2}$, and surface $\mathrm{pH}$ are well-represented in this configuration of CESM relative to observations ${ }^{27,28}$. However, the coarser grid resolution suppresses variability in surface $\mathrm{pH}$. In turn, the annual surface $\mathrm{pH}$ anomalies being predicted are smaller than 0.01 units (Fig. 1B), but these relatively small anomalies are associated with large 
286 fluctuations in other environmentally relevant variables, such as the aragonite saturation

287 state, which varies on the order of 0.1 units (Fig. S6D). In spite of the relatively small

288 target anomalies being predicted, CESM-DPLE forecast error (as measured by the

289 NMAE) falls within the spread of the historical surface pH variability (Fig. 4B and 6). In

290 this study, we only highlight predictability in annual averages of surface $\mathrm{pH}$, since

291 predictability at annual resolution is much higher than that of monthly resolution.

292 However, we do find significant predictability of surface $\mathrm{pH}$ anomalies over forecasts of

293 persistence and external forcing through June of the upwelling season following

294 initialization, and into April of the following upwelling season (Fig. S7). We focus on

295 assessing predictability in surface $\mathrm{pH}$ after removing the ocean acidification trend to

296 highlight the role of initialization in engendering predictability. Our results are similar if

297 we conduct the analysis on trended surface pH (Fig. S8). Lastly, in assessing predictive

298 skill, we are challenged by the limited coverage of gridded surface $\mathrm{pH}$ observations.

299 While the observational product used in this study spans 1990-2017, the observational

300 data for atmospheric $\mathrm{CO}_{2}$ used to force the reconstruction ended in 2005, after which

301 point a smooth scenario-based projection was used (Fig. S1A). This causes a drop-off

302 in the ability of the reconstruction to replicate observed surface $\mathrm{pH}$ anomalies (Fig.

303 S1B). Thus, we only assess skill over the 1990-2005 period, limiting our degrees of

304 freedom for statistical significance.

305 Our results demonstrate for the first time the potential for an initialized ESM to 306 retrospectively predict surface $\mathrm{pH}$ multiple years in advance in a complex, sensitive, and

307 economically important oceanic region. Although these forecasts cannot aid directly in 
308 the management of coastal fisheries at this spatial resolution, our results demonstrate

309 the feasibility of making skillful surface $\mathrm{pH}$ predictions on multiannual to decadal

310 timescales. Further, global initialized ESM forecasts can be used as boundary

311 conditions to improve existing regional biogeochemical forecasting and to extend their

312 lead times. While our study highlights CESM-DPLE's ability to predict surface $\mathrm{pH}$

313 anomalies, other ocean acidification parameters-such as calcium carbonate saturation

314 states - can be expected to be predictable, due to their common dependence on

315 variability in dissolved $\mathrm{CO}_{2}$. By detrending our simulated and observational products

316 prior to analysis, we show that we have the potential to predict interannual variations in

317 surface $\mathrm{pH}$. As the ocean acidification signal dominates in this region over decadal

318 timescales, multiyear predictions of surface $\mathrm{pH}$ variability could aid in forecasting the

319 acceleration or slowdown of ocean acidification in the CCS.

320 Methods

$321 \quad$ Model simulations

322 The Community Earth System Model Decadal Prediction Large Ensemble 22 (CESM-

323 DPLE) is based on CESM, version 1.1, and uses the same code base, component

324 model configurations (Table S2), and historical and projected radiative forcing as that

325 used in its counterpart, the CESM Large Ensemble 24 (CESM-LE). This includes

326 historical radiative forcing (with volcanic aerosols) through 2005 and projected radiative

327 forcing (including greenhouse and short-lived gases and aerosols) from 2006 onward.

328 The main difference between the two experiments is that CESM-DPLE is re-initialized

329 annually to generate forecast ensembles (see next paragraph for details), while CESM- 
330 LE is only initialized once. We follow the convention of the decadal prediction

331 community $\underline{21}$ and refer to the former as the "initialized" ensemble and the latter as the

332 "uninitialized" ensemble. Because CESM-DPLE and CESM-LE have an identical code

333 base and boundary conditions, the two ensembles can be compared directly to one

334 another to isolate the relative influence of re-initialization and external forcing on

335 hindcast predictability and skill.

CESM-DPLE was generated via full-field initialization each year on November $1^{\text {st }}$

337 from 1954 to 2017 , for a total of 64 initialization dates $\stackrel{21}{ }$. An ensemble of 40 forecast

338 members was created by making Gaussian perturbations to the initial atmospheric

339 temperature field (order $10^{-14} \mathrm{~K}$ ) at each grid cell. Ensemble spread in all other fields

340 and model components developed as a result of the spread in the atmospheric state.

341 Each member was integrated forward from each initialization for 122 months, resulting

342 in approximately 26,000 global fully coupled simulation years, costing roughly 50 million

343 core hours to compute. The atmosphere and land components were initialized from the

344 November $1^{\text {st }}$ restart files of a single arbitrary member of CESM-LE (ensemble member

$34534)^{36}$. The atmosphere component is the Community Atmosphere Model, version 5

346 (CAM5) with a finite-volume dynamical core at nominal $1_{-}^{\circ}$ resolution and 30 vertical

347 levels $\mathbf{2 1 , 3 7}$. Details on the land component can be found in Table S2.

348 The ocean (including biogeochemistry) and sea ice model components in CESM-

349 DPLE were re-initialized from the November $1^{\text {st }}$ restart files of a forced ocean-sea ice

350 reconstruction (referred to as the "reconstruction"; see following section for configuration

351 details). The ocean biogeochemical model used in all CESM simulations in this study is 
352 the Biogeochemical Elemental Cycling (BEC) model, which contains three

353 phytoplankton functional types (diatoms, diazotrophs, and a small calcifying

354 phytoplankton class), explicitly simulates seawater carbonate chemistry, and tracks the

355 cycling of $\mathrm{C}, \mathrm{N}, \mathrm{P}, \mathrm{Fe}, \mathrm{Si}$, and $\mathrm{O} \underline{38.39}$. Note that the ocean biogeochemistry and

356 simulated atmospheric $\mathrm{CO}_{2}$ concentration are diagnostic, such that there is no feedback

357 onto the simulated physical climateㄴ⒈ Further details on drift adjustment and anomaly

358 generation can be found in the supplemental materials.

The reconstruction simulation was run from 1948-2017 with active ocean

360 (physics and biogeochemistry) and sea ice model components from CESM, version 1.1, 361 with identical spatial resolutions as the fully coupled CESM-DPLE and CESM-LE (Table 362 S2). The ocean and sea ice components were forced by a modified version of the

363 Coordinated Ocean-Ice Reference Experiment (CORE) with interannual forcing ${ }^{40,41}$, 364 which provides momentum, freshwater, and buoyancy fluxes between the air-sea and 365 air-ice interfaces. CORE winds were used globally, save for the tropical band (30S$36630 \mathrm{~N}$ ), where NOAA Twentieth Century Reanalysis, version $2^{42}$ winds (from 1948-2010) 367 and adjusted Japanese 55-year Reanalysis Project ${ }^{43}$ winds (through 2017) were used to

368 correct a spurious trend in the zonal equatorial Pacific sea surface temperature (SST)

369 gradient $^{21}$. No direct assimilation of ocean or sea ice observations was used in the

370 reconstruction; thus, any faithful reproduction of ocean and sea ice climatology or

371 variability is due mainly to the atmospheric reanalysis that drives the simulation ${ }^{21}$.

372 Observational product 
373 We compare initialized forecasts of surface $\mathrm{pH}$ to the Japanese Meteorological Agency

374 (JMA) Ocean $\mathrm{CO}_{2}$ Map product ${ }^{25,44}$, which provides monthly estimates of $\mathrm{pH}$ from

375 1990-2017 over a $1^{\circ} \times 1^{\circ}$ global grid. Here, we describe the key steps followed by the

376 authors of the JMA product to derive their surface $\mathrm{pH}$ estimates. Surface $\mathrm{pH}$ was

377 computed diagnostically with a carbonate system solver, using estimated surface

378 alkalinity and $\mathrm{pCO}_{2}$ as inputs. To compute gridded alkalinity, the ocean was divided into

379 five regions, where empirical relationships were derived for in situ alkalinity as a function

380 of sea surface height (SSH) and sea surface salinity ${ }^{25}$ (SSS). Gridded observations of

$381 \mathrm{SSH}$ and SSS (independent of the in situ observations) were then input into the

382 empirical equations to derive gridded surface alkalinity. Gridded surface $\mathrm{pCO}_{2}$ was

383 computed through a multistep process. First, the ocean was divided into 44 regions and

384 relationships between in situ $\mathrm{pCO}_{2}$ and in situ SST, SSS, and Chl-a were derived by

385 multiple linear regressions in each region for one to three of the variables ${ }^{44}$. The gridded

$386 \mathrm{pCO}_{2}$ product was then derived by applying these functions to independent gridded

387 observations of SST, SSS, and Chl-a. There are no uncertainty estimates available for

388 the $\mathrm{pH}$ product, but the authors report a root mean square error (gridded estimate

389 compared to in situ observations) of $10-20 \mu$ atm for $\mathrm{pCO}_{2}$ in the northern hemisphere

390 mid-latitudes and $8.1 \mu \mathrm{mol} \mathrm{kg}{ }^{-1}$ for surface alkalinity relative to the PACIFICA

391 campaign ${ }^{25,44}$. Note that the global average JMA surface $\mathrm{pH}$ is within the uncertainty of

392 the SOCAT-based estimate for all years (Fig. S9). Further details on the datasets used

393 in deriving their product can be found in Takatani et al. 2014 and lida et al. 2015.

394 Statistical analysis 
395 We use deterministic metrics to compare the ensemble mean retrospective forecasts to 396 one or both of the following reference forecasts: (1) a persistence forecast, and (2) the 397 uninitialized CESM-LE ensemble mean forecast. A comparison of the initialized forecast 398 to the persistence forecast shows the utility of our initialized forecasting system over a 399 simple, low-cost forecasting method; a comparison of the initialized forecast to the 400 uninitialized forecast shows the utility of initializations (rather than external forcing) in 401 lending predictability to the variable of interest. The persistence forecast assumes that anomalies from each initialization year persist into all following lead years (or months) ${ }^{45}$.

403 The uninitialized forecast compares the CESM-LE ensemble mean anomalies to the 404 verification data (model reconstruction or observations) over the same window as the 405 initialized forecasting system ${ }^{21}$. Unless otherwise noted, forecasts are analyzed at 406 annual resolution. This corresponds to the January-December average following the 407 November $1^{\text {st }}$ initialization. In turn, lead year "one" truly covers lead months $3-14$. When 408 considering monthly predictions, lead month "one" corresponds to the November $1^{\text {st }}$ $40930^{\text {th }}$ average following initialization.

We compute the anomaly correlation coefficient (ACC) via a Pearson product-

411 moment correlation to quantify the linear association between predicted and target 412 anomalies (where the target is either the model reconstruction or the observational 413 product). If the predictions perfectly match the sign and phase of the anomalies, the 414 ACC has a maximum value of 1 . If they are exactly out of phase, it has a minimum 415 value of -1 . The ACC is a function of lead time ${ }^{10,46}$ : 


$$
\operatorname{ACC}(\tau)=\frac{\left(\sum_{\propto=1}^{N}\left(F_{\propto}^{\prime}(\tau) \times O_{\alpha+\tau}^{\prime}\right)\right)}{\sqrt{\sum_{\propto=1}^{N} F_{\propto}^{\prime}(\tau)^{2} \sum_{\propto=1}^{N} O_{\alpha+\tau}^{\prime}{ }^{2}}}
$$

Where $F^{\prime}$ is the forecast anomaly, $O^{\prime}$ is the verification field anomaly, and the

ACC is calculated over the initializations spanning 1954-2017 ( $N=64)$ relative to the reconstruction and CESM-LE, and over initializations covering 1990-2005 ( $\mathrm{N}=16)$ relative to the JMA observational product. We quantify statistical significance in the ACC using a $t$ test at the $95 \%$ confidence level with the null hypothesis that the two time series being compared are uncorrelated. We follow the methodology of Bretherton et al. $(1999)^{47}$, using the effective sample size in $t$ tests to account for autocorrelation in the two time series being correlated:

$$
N_{e f f}=N\left(\frac{1-\rho_{1} \rho_{2}}{1+\rho_{1} \rho_{2}}\right)
$$

Where $\mathrm{N}$ is the true sample size and $\rho_{1}$ and $\rho_{2}$ are the lag 1 autocorrelation coefficients for the forecast and verification data. We assess statistical significance between two ACCs (e.g., between that of the initialized forecast and a simple persistence forecast for the same lead time) using a $z$ test at the $95 \%$ confidence level with the null hypothesis that the two correlation coefficients are not different.

To quantify the magnitude of forecast error, or the accuracy in our forecasts, we use the normalized mean absolute error ${ }^{46}$ (NMAE), which is the MAE normalized by the interannual standard deviation of the verification data. The NMAE is 0 for perfect forecasts, less than 1 when the forecast error falls within the variability of the verification data, and increases as the forecast error surpasses the variability of the verification 
436 data. MAE is used instead of bias metrics such as the root mean square error (RMSE),

437 as it is a more accurate assessment of bias in climate simulations ${ }^{48}$.

$$
\operatorname{NMAE}(\tau)=\frac{1}{N} \sum_{\propto=1}^{N} \frac{\left|F_{\propto}^{\prime}(\tau)-O_{\alpha+\tau}^{\prime}\right|}{\sigma_{O^{\prime}}(\tau)}
$$

Where $\mathrm{N}$ is the number of initializations and $\sigma_{O^{\prime}}$ is the standard deviation of the verification data over the verification window.

\section{Linear Decompositions}

We follow Lovenduski et al. (2019) ${ }^{17}$ to convert predictability in $\mathrm{pH}$ driver variables (SST, SSS, salinity-normalized dissolved inorganic carbon (SDIC), and salinity-normalized alkalinity (sALK)) to common $\mathrm{pH}$ units:

$$
r_{x} \cdot \frac{d p H}{d x} \cdot \sigma_{x}
$$

Where $r_{x}$ is the ACC between anomalies in driver variable $x$ and target

anomalies, $\frac{d p H}{d x}$ is the linear sensitivity of $\mathrm{pH}$ to the driver variable, and $\sigma_{x}$ is the standard deviation of driver variable anomalies in the reconstruction.

We use a linear Taylor expansion to quantify the relative contribution of variability in environmental drivers to total surface $\mathrm{pH}$ variability in the $\mathrm{CCS}^{28,49}$ :

$$
p H^{\prime}=\frac{d p H}{d T} T^{\prime}+\frac{d p H}{d S} S^{\prime}+\frac{d p H}{d D I C} s D I C^{\prime}+\frac{d p H}{d A L K} s A L K^{\prime}+\text { residual }
$$

Where primes denote annual average anomalies after removing a second-order polynomial fit, and $\frac{d p H}{d x}$ the linear sensitivity of $\mathrm{pH}$ to the driver variable $x$. Residual variability is due to freshwater dilution effects, higher-order terms excluded in the linear expansion, and cross-derivative terms ${ }^{28}$. Sensitivities were computed using the 
carbonate system solver, CO2SYS. For example, $\frac{d p H}{d T}$ was computed by varying SST by its seasonal range in the CCS in the model reconstruction while holding DIC, alkalinity, and salinity constant at their mean values in the CCS. A linear slope was then fit to the resulting change in surface $\mathrm{pH}$ over this range.

\section{Acknowledgements}

The CESM project is supported primarily by the National Science Foundation (NSF). This material is based upon work supported by the National Center for Atmospheric Research, which is a major facility sponsored by the NSF under Cooperative Agreement No. 1852977. Computing and data storage resources, including the Cheyenne supercomputer (doi: 10.5065/D6RX99HX), were provided by the Computational and Information Systems Laboratory (CISL) at NCAR. The Department of Energy's Computational Science Graduate Fellowship supported RXB throughout this study (DEFG02-97ER25308). NSL and RXB are grateful for support from the NSF (OCE1752724). RXB acknowledges Aaron Spring for his contributions to analysis through collaborative development of the climpred package (see additional information) as well as Samantha Siedlecki, Michael Jacox, and Michael Alexander for suggestions during the analysis phase of the project.

\section{Author contributions}

RXB and NSL designed the study. RXB analyzed the data, prepared figures and tables, and wrote the paper. SGY and KL coordinated and ran CESM-DPLE and FOSI simulations. NSL, SGY, MCL, and KL provided invaluable feedback throughout the study and reviewed the manuscript. 


\section{Data Availability}

Output from the CESM-DPLE and reconstruction can be downloaded through the Earth System Grid Federation

(https://www.earthsystemgrid.org/dataset/ucar.cgd.ccsm4.CESM1-CAM5-DP.html). The JMA Ocean $\mathrm{CO}_{2}$ map product can be downloaded online at https://www.data.jma.go.jp/gmd/kaiyou/english/co2 flux/co2 flux data en.html.

\section{Code Availability}

Analysis was performed using climpred, an open source python package developed by the lead author for analyzing initialized forecast models. Documentation is available at https://climpred.readthedocs.io. Post-processed model output and observations as well as the code used to create all figures in this study will be made available on Zenodo and Github (https://github.com/bradyrx/ncomms $\mathrm{pH}$ predictability) following acceptance and publication of this manuscript.

\section{Competing Interests}

The authors of this study are unaware of any competing interests.

\section{References}

1. Doney, S. C., Fabry, V. J., Feely, R. A. \& Kleypas, J. A. Ocean Acidification: The Other $\mathrm{CO}_{2}$ Problem. Annu. Rev. Mar. Sci. 1, 169-192 (2009).

2. Gruber, N. et al. Rapid Progression of Ocean Acidification in the California Current System. Science 337, 220-223 (2012).

3. Huyer, A. Coastal upwelling in the California current system. Prog. Oceanogr. 12, 259-284 (1983). 
4. Pauly, D. \& Christensen, V. Primary production required to sustain global fisheries. Nature 374, 255-257 (1995).

5. Feely, R. A., Sabine, C. L., Hernandez-Ayon, J. M., Ianson, D. \& Hales, B. Evidence for Upwelling of Corrosive 'Acidified' Water onto the Continental Shelf. Science 320, 1490-1492 (2008).

6. Bednaršek, N. et al. Limacina helicina shell dissolution as an indicator of declining habitat suitability owing to ocean acidification in the California Current Ecosystem. Proc R Soc B 281, 20140123 (2014).

7. Bednaršek, N. et al. Exposure history determines pteropod vulnerability to ocean acidification along the US West Coast. Sci. Rep. 7, (2017).

8. Fisheries economics of the United States 2015. (2017).

9. Jacox, M. G., Alexander, M. A., Stock, C. A. \& Hervieux, G. On the skill of seasonal sea surface temperature forecasts in the California Current System and its connection to ENSO variability. Clim. Dyn. (2017) doi:10.1007/s00382-017-3608-y.

10. Hervieux, G. et al. More reliable coastal SST forecasts from the North American multimodel ensemble. Clim. Dyn. (2017) doi:10.1007/s00382-017-3652-7.

11. Stock, C. A. et al. Seasonal sea surface temperature anomaly prediction for coastal ecosystems. Prog. Oceanogr. 137, 219-236 (2015).

12. Siedlecki, S. A. et al. Experiments with Seasonal Forecasts of ocean conditions for the Northern region of the California Current upwelling system. Sci. Rep. 6, (2016). 
13. Park, J.-Y., Stock, C. A., Dunne, J. P., Yang, X. \& Rosati, A. Seasonal to multiannual marine ecosystem prediction with a global Earth system model. Science 365, 284-288 (2019).

14. Séférian, R. et al. Multiyear predictability of tropical marine productivity. Proc. Natl. Acad. Sci. 111, 11646-11651 (2014).

15. Li, H., llyina, T., Müller, W. A. \& Sienz, F. Decadal predictions of the North Atlantic $\mathrm{CO}_{2}$ uptake. Nat. Commun. 7, (2016).

16. Séférian, R., Berthet, S. \& Chevallier, M. Assessing the Decadal Predictability of Land and Ocean Carbon Uptake. Geophys. Res. Lett. 45, 2455-2466 (2018).

17. Lovenduski, N. S., Yeager, S. G., Lindsay, K. \& Long, M. C. Predicting near-term variability in ocean carbon uptake. Earth Syst. Dyn. 10, 45-57 (2019).

18. Li, H., llyina, T., Müller, W. A. \& Landschützer, P. Predicting the variable ocean carbon sink. Sci. Adv. 5, eaav6471 (2019).

19. Tommasi, D. et al. Managing living marine resources in a dynamic environment: The role of seasonal to decadal climate forecasts. Prog. Oceanogr. 152, 15-49 (2017).

20. Bonan, G. B. \& Doney, S. C. Climate, ecosystems, and planetary futures: The challenge to predict life in Earth system models. Science 359, eaam8328 (2018).

21. Yeager, S. G. et al. Predicting Near-Term Changes in the Earth System: A Large Ensemble of Initialized Decadal Prediction Simulations Using the Community Earth System Model. Bull. Am. Meteorol. Soc. 99, 1867-1886 (2018).

22. Meehl, G. A. et al. Decadal Climate Prediction: An Update from the Trenches. Bull. Am. Meteorol. Soc. 95, 243-267 (2014). 
23. Branstator, G. \& Teng, H. Two Limits of Initial-Value Decadal Predictability in a CGCM. J. Clim. 23, 6292-6311 (2010).

24. Kay, J. E. et al. The Community Earth System Model (CESM) Large Ensemble Project: A Community Resource for Studying Climate Change in the Presence of Internal Climate Variability. Bull. Am. Meteorol. Soc. 96, 1333-1349 (2015).

25. Takatani, Y. et al. Relationships between total alkalinity in surface water and sea surface dynamic height in the Pacific Ocean. J. Geophys. Res. Oceans 119, 28062814 (2014).

26. lida, Y. et al. Trends in $\mathrm{pCO}_{2}$ and sea-air $\mathrm{CO}_{2}$ flux over the global open oceans for the last two decades. J. Oceanogr. 71, 637-661 (2015).

27. Brady, R. X., Alexander, M. A., Lovenduski, N. S. \& Rykaczewski, R. R. Emergent anthropogenic trends in California Current upwelling. Geophys. Res. Lett. 44, 2017GL072945 (2017).

28. Brady, R. X., Lovenduski, N. S., Alexander, M. A., Jacox, M. \& Gruber, N. On the role of climate modes in modulating the air-sea $\mathrm{CO}_{2}$ fluxes in eastern boundary upwelling systems. Biogeosciences 16, 329-346 (2019).

29. Landschützer, P. et al. A neural network-based estimate of the seasonal to interannual variability of the Atlantic Ocean carbon sink. Biogeosciences 10, 7793-7815 (2013).

30. Laruelle, G. G. et al. Global high-resolution monthly $\mathrm{pCO}_{2}$ climatology for the coastal ocean derived from neural network interpolation. Biogeosciences 14, 4545-4561 (2017). 
522

523

524

525

526

527

528

529

530

531

532

31. Hauri, C. et al. Spatiotemporal variability and long-term trends of ocean acidification in the California Current System. Biogeosciences 10, 193-216 (2013).

32. Pozo Buil, M. \& Di Lorenzo, E. Decadal dynamics and predictability of oxygen and subsurface tracers in the California Current System. Geophys. Res. Lett. 44, 42044213 (2017).

33. Bograd, S. J., Schroeder, I. D. \& Jacox, M. G. A Water Mass History of the Southern California Current System. Geophys. Res. Lett. 2019GL082685 (2019) doi:10.1029/2019GL082685.

34. Gent, P. R. \& Mcwilliams, J. C. Isopycnal Mixing in Ocean Circulation Models. J. Phys. Oceanogr. 20, 150-155 (1990).

35. Gruber, N. et al. Eddy-induced reduction of biological production in eastern boundary upwelling systems. Nat. Geosci. 4, 787-792 (2011).

36. Lovenduski, N. S., Bonan, G. B., Yeager, S. G., Lindsay, K. \& Lombardozzi, D. L. High predictability of terrestrial carbon fluxes from an initialized decadal prediction system. Environ. Res. Lett. 14, 124074 (2019).

37. Hurrell, J. W. et al. The Community Earth System Model: A Framework for Collaborative Research. Bull. Am. Meteorol. Soc. 94, 1339-1360 (2013).

38. Moore, J. K., Lindsay, K., Doney, S. C., Long, M. C. \& Misumi, K. Marine Ecosystem Dynamics and Biogeochemical Cycling in the Community Earth System Model [CESM1(BGC)]: Comparison of the 1990s with the 2090s under the RCP4.5 and RCP8.5 Scenarios. J. Clim. 26, 9291-9312 (2013). 
39. Lindsay, K. et al. Preindustrial-Control and Twentieth-Century Carbon Cycle Experiments with the Earth System Model CESM1(BGC). J. Clim. 27, 8981-9005 (2014).

40. Griffies, S. M. et al. Coordinated Ocean-ice Reference Experiments (COREs). Ocean Model. 26, 1-46 (2009).

41. Large, W. G. \& Yeager, S. G. The global climatology of an interannually varying airsea flux data set. Clim. Dyn. 33, 341-364 (2009).

42. Compo, G. P. et al. The Twentieth Century Reanalysis Project. Q. J. R. Meteorol. Soc. 137, 1-28 (2011).

43. Tsujino, H. et al. JRA-55 based surface dataset for driving ocean-sea-ice models (JRA55-do). Ocean Model. 130, 79-139 (2018).

44. lida, $\mathrm{Y}$. et al. Trends in $\mathrm{pCO}_{2}$ and sea-air $\mathrm{CO}_{2}$ flux over the global open oceans for the last two decades. J. Oceanogr. 71, 637-661 (2015).

45. Van den Dool, H., Cpc, P. S. \& others. Empirical methods in short-term climate prediction. (Oxford University Press, 2007).

46. Jolliffe, I. T. \& Stephenson, D. B. Forecast verification: a practitioner's guide in atmospheric science. (John Wiley \& Sons, 2012).

47. Bretherton, C. S., Widmann, M., Dymnikov, V. P., Wallace, J. M. \& Bladé, I. The Effective Number of Spatial Degrees of Freedom of a Time-Varying Field. J. Clim. 12, 1990-2009 (1999). 
563 48. Willmott, C. \& Matsuura, K. Advantages of the mean absolute error (MAE) over the 564 root mean square error (RMSE) in assessing average model performance. Clim. Res. 30, 79-82 (2005).

566 49. Lovenduski, N. S., Gruber, N., Doney, S. C. \& Lima, I. D. Enhanced CO2 outgassing 567 in the Southern Ocean from a positive phase of the Southern Annular Mode. Glob. $568 \quad$ Biogeochem. Cycles 21, GB2026 (2007). 